\title{
МНЕМОФУНКЦИИ И НЕКОТОРЫЕ ИХ ПРИЛОЖЕНИЯ
}

\author{
А.Б.АНТОНЕВИЧ, Н.Я.РАДЫНО, Я.В.РАДЫНО
}

\author{
Белорусский государственный университет, \\ механико-- математический факультет, \\ кафедра функционального анализа \\ 200050 г.Минск, пр. Скорины 4. \\ Институт математики Академии Наук Беларуси, \\ отдел стохастического анальза, \\ 220072 г.Минск, ул. Сурганова 11.
}

Работа выполнена при финансовой поддержке Фонда фундаментањньхх исследований Республики Беларусь и мехдународного фонда Сороса

Веденше. Теория распределений привела к существенному прогрессу в ряде математических писциплин типа линейных дифференциальных уравнений с частными произвопњыми и линейной математической физики. Јоран Шварц показал, что невозможно определить ассоциативное умнохение распределенй [1]. Этот факт является препятствием к испольованию распределений в теории нелинейньх уравнений и теории уравнений с разрыпныпи коэффициентами. В частности, это приводит к невозможности придать смысл таким объектам, как $\delta^{2}, \delta^{\prime} \delta(\delta-$ функщия Дирака), которые широко используются, например, в квантово теории поля.

Рассматриваемая проблема очень естественна и имеет многочисленные применения.Поэтому она привлекла к себе внимание многочисленных авторов сразу же после создания теории распределений.

О6зор работ по этон тематике имеется, например, в [2]. В данной работе излагаются основные идеи, исполыземые при решении проблемы умножения распределений, а также указываются некоторые направления приложений.

Нетривиальность этон проблемы видна на следующем примере. Пусть $u, v \in D^{\prime}(\mathbf{R})$ и пусть $u_{n}$, $v_{n} \in C^{\infty}(\mathbf{R})$ такие, что, $u_{n} \rightarrow u, v_{n} \rightarrow v$ в $D^{\prime}(\mathbf{R})$. Последоватељьость произведений $u_{n} v_{n}$ может не сходится в $D^{\prime}(\mathbf{R})$, а может иметь предел, зависяший от выбора последовательностей $u_{n}$ и $v_{n}$. Например, для любого $a \in C^{\infty}(\mathbf{R})$ последовательность $u_{n}(\boldsymbol{x})=a(\boldsymbol{x}) \sin (\boldsymbol{n} \boldsymbol{x})$ сходится к нулю в $D^{\prime}(\mathbf{R})$, в то время как последовательность $u_{n}^{2}(x)$ сходится к $a^{2}(x) / 2$.

Стақдартны подход $\mathrm{k}$ решению рассматриваемой проблемы основывается на определенном выборе последовательностен $u_{n}$ и $v_{n}$. В этом случае определить произведение можно дия некоторых пар $u_{n}$ и $v_{n}$. Друтой подход основан на введении вместо распределении новых объектов, которые с одной стороны, обладают основными свонистами распределений, и, с другой стороны, допускают хорошо определенное умножение, то есть образуют ассоциативную алгебру.

Различные варианты введения таких обьектов были даны в работах Б. Дамянова и Хр. Христова [3], В. К. Иванова [4], Ж. Коломбо [5] и Ю. Егорова [2]. Общая схема построения таких алге6р такого типа была предложена авторами в работе [6]. Наш метод позволяет строить различные алгебры с заданными дополнительными свойствами.

Заметим, что этот метод позволяет дать решение еще оцной задачи: о задании всюду определеннон свертки.

Некоторые из уже известных конструкций, например, нестандартное расигирение, являются частными случаями наших построений.

2. Постановка задани и обпая схема репения. Пусть $E^{\prime}$ некоторое пространство распределений и $A$ плотно вложенная в $E^{\prime}$ алтебра бесконечно дифференцируемых функцин. Требуется построить 
новую алгебру $G$ и линейное вложение $j: E \rightarrow G$ такие, что $A$ влохена в $G$ как подалгебра, то есть

(1)

$$
j(a b)=j(a) j(b), \quad a, b \in A
$$

Имеется определенная тонкость в постановке проблемы. В самом деле, пример Л.Шварца, показывает, что нельзя вложить пространство $D^{\prime}(\mathbf{R})$ в алгебру, удовлетворяющую условию: $j(a u)=j(a) j(u)$ для $a \in A, u \in E^{\prime}$, если $E^{\prime}=D^{\prime}(\mathbf{R}), \quad A=C^{\infty}(\mathbf{R})$. Поэтому мы можем требоватъ сохранения умножения только для элементов алгебры глапхих функции.

Нижеприведенные построения не испоӭьзуют того факта, что пространство $E^{\prime}$ состоит из распределений и имеют более общий характер. Исходными объектами наших построений являются:

а) отделимое локально выпуклое пространство $E^{\prime}$ (для элементов которого требуется определитъ произведение);

6) локально выпуклая алгебра $A$ непрерывно и плотно вложенная в $E^{\prime}$;

с) семейство непрерывнгх линейньг операторов $R_{\phi, \tau}: E \rightarrow A$, где $\phi \in \Phi, \tau \in I$; (Ф -фиксированное множество, $I$ - множество с заданным на нем фильтром $F$ такое, что для каждого фиксированного $\phi, R_{\phi, \tau}(u) \rightarrow u$ в топологии $E^{\prime}$ по фиљтру $F$ для кахдого $u \in E^{\prime}$. Для фиксированного $\phi$, семейство $R_{\phi, \tau}(u) \in I$, назвается методом регуляризации элементов $E^{\prime}$ элементами $A$.

Обозначим через $G$-мнохество всех отобрахений из $\Phi \times I$ в $A$. Это множество является алтеброй и пространство $E^{\prime}$ вкладывается в $\tilde{G}$ с помощью отобрахения $j_{0}: u \rightarrow R_{\phi, \tau}(u)$.

В ряде ситуаций эта естественная и "тривиатьная" конструкция вложения $E^{\prime}$ в алтебру позволяет получать содержательные результаты. Однако поставленной выше задачи она не решает, так как равенство (1) не выполняется. Кроме того, алгебра $G$ чрезвьчайно обширна и в конкретных задачах

оказывяется, что различные ее элементы описывают одно и то хе физическое состояние и потому они дольхы быть отохдествлены.

Предлагаемая конструкция закпочается в следуюшем. В алгебре $G$ выделяется подалгебра $G_{M}$ и в ней некоторын идеал $N$. Искомым объектом является фактор-алгебра $G_{M} / N$. Таким образом, конструкция сводится к выбору подходящей пары $\left(G_{M} / N\right)$.

Два элемента $f$ и $g$ из называются слабо эквивалентными, если $f(\phi, \tau)-g(\phi, \tau) \rightarrow 0$ в $E^{\prime}$ по $F$ для любого $\phi \in \Phi$. Мнохество $N_{0}$ всех элементов слабо эквивалентных нулю не является идеалом и даже подалгеброй в $G$. Поэтому отношение слабой эквивалентности, естественное в других задачах, не решгает поставленной выше проблемы.

Теорема 1. Предполозим, ито подалгебра $G_{M}$ и идеал $N$ удовлетворяют следуюиим условик: i) для каждого $u \in E^{\prime}$ элемент $R_{\phi, \tau} \in G_{M}$;

ii) $N \subset N_{0}$;

iii) элемекты вида $R_{\phi, \tau}(a b)-R_{\phi, \tau}(a) R_{\phi, \tau}(b)$ принадлежат $N$ для любьх $a, b \in A$.

Тогда пространство $E^{\prime}$ вкладввается в фактор-алгебру $G=G_{M} / N$ как подпространство, а $A$ как подалгера. Если $G_{M}$ и $N$ инвариантны относительно некоторого линейного непрерыяного операmоро $L: A \rightarrow A$, то тогда этот оператор может быть естественным образом продолжен до опероmopo в алгебре $G$

Поскольку услочие (ii) ограничивает идеал $N$ сверху, а условие (iii) ограничивает его снизу, то существование идеала, удовлетворяющего условиям (ii) и (iii), не очевидно. Явное описание зависит от выбора семейства методов регуляризации. Спосо6 выбора подалгебры $G_{M}$ и идеала $N$ может быть предложен с помошью топологии алгебры $A$ 
Предположим, что топология на $A$ определена с помощью мультипликативной системы полунорм $\left(p_{\alpha}\right), \alpha \in \Lambda$, то есть полунорм, удовлетворяющих условию

$$
p_{\alpha}(a b) \leq C_{\alpha} p_{\alpha}(a) p_{\alpha}(b)
$$

Скалярные функции $p_{\alpha}\left(R_{\phi, \tau}(u)\right), \quad u \in E^{\prime}$ датот типичны рост по $\tau$ элементов из $G_{M}$, а функиии $p_{\alpha}\left(R_{\phi, \tau}(a b)\right)-R_{\phi, \tau}(a) R_{\phi, \tau}(b), \quad a, b \in A$ описывают типичное убывание по $\tau$ элементов из $N$

Пусть $H_{\alpha}$-атебра скалмнньх функии, определегных на $\Phi \times I$, порождена функиями вида $p_{\alpha}\left(R_{\phi, \tau}(u)\right), \quad u \in E^{\prime}$ и пусть $N_{\alpha}$-апгебра скальрных функций, заданныг на $\Phi \times I$ порождена функииями вида

$$
p_{\alpha}\left(R_{\phi, \tau}(a b)\right)-R_{\phi, \tau}(a) R_{\phi, \tau}(b), \quad a, b \in A
$$

Следующая теорема позволяет свести анализ к более простому слугаю скалярных функиии.

Теоремя 2. Пусть для хахдого $\alpha \in \Lambda$ алтебра $N_{\alpha}$ является идеалом в $H_{\alpha}$ Тогда множества\}

$$
\begin{aligned}
& G_{M}(A)=\left\{f \in \tilde{G}: \forall h \exists \alpha \in H_{\alpha}, p_{\alpha}(f(\phi, \tau)) \leq h(\phi, \tau)\right\}, \\
& N(A)=\left\{f \in \tilde{G}: \forall \alpha \exists h \in N_{\alpha}, p_{\alpha}(f(\phi, \tau)) \leq h(\phi, \tau)\right\}
\end{aligned}
$$

удовлетворяют условиям теоремы 1 и фактор-алгебра $G=G_{M}(A) / N(A)$ дает решение проблемы.

3. Алгебра Коломбо. Перөы пример пары $\left(G_{N}, N\right)$ был построен Ж. Коломбо.

Пусть $E^{\prime}=D^{\prime}(\mathbf{R}), \quad A=C^{\infty}(\mathbf{R})$, и $\Phi$-суетное мнохество

$$
\Phi=\left\{\phi_{1}, \phi_{2}, \ldots\right\}, \phi \in D(\mathbf{R}) \text {, }
$$

$$
\int \phi_{q}(x) d x=1, \quad \int x^{j} \phi_{q}(x) d x=0, \quad j=1,2, \ldots, q .
$$

Каждая из функший $\phi_{q}$ определяет метод регуляризации формулой

$$
R_{q, \tau}(u)=u * \phi_{q, \tau}=u_{q, \tau}, \quad \phi_{q, \tau}(x)=\frac{1}{\tau} \phi_{q}\left(\frac{x}{\tau}\right), \quad \tau \in l=(0,1) \subset \mathbf{R}
$$

На алтебре $C^{\infty}(\mathbf{R})$ существует мультипликативная система полунорм:

$$
p_{k, n}(u)=\sum_{j=0}^{k} \max _{|x| \leq n} \frac{u^{(j)}(x)}{k !}, \quad k=1,2, \ldots, n=1,2, \ldots
$$

Функции $p_{k, n}\left(u_{q, \tau}\right)$ махорируются некоторон степенью $1 / \tau$, когда $\tau \rightarrow 0$. Поэтому естественно определить

$$
G_{M}=\left\{f_{q, \tau}(x): \forall k, n \exists m, c>0: p_{k, n}\left(f_{q, \tau}\right) \leq \frac{c}{\tau^{m}}\right\} .
$$

Функиии вида $p_{k, n}\left((a b)_{q, \tau}-a_{q, \tau} b_{q, \tau}\right), a, b \in C^{\infty}$ (R) имеют степенное убывание по $\tau$ при $\tau \rightarrow 0$ Для каждого фиксированного $q$ множество функцин со степенпым убыванием не является, вообще говоря, идеалом в алгебре функций, растущих с некоторой степенью $\tau$. Ж.Коломбо заметил и испопъзовал тот факт, что скорость убывания этих функций увеличивается с увеличением $\tau$. Поэтому множество

$$
N=\left\{f_{q, \tau}(x): \forall k, n \exists q_{0} c_{q}: P_{k, n}\left(f_{q, \tau}\right) \leq c_{q} \tau^{q-q_{0}}\right\}
$$

является идеалом в $G_{M}$ и удовлетворяет условиям теорем 1 и 2. Фактор-алтебра $G=G_{N} / N$ является модификацией атгебры Коломбо "новых обобщегцых функций". 
Отметим, что вложение пространства $D^{\prime}(\mathbf{R})$ в $G$ зависит от выбора последовательности $\left(\phi_{q}\right)$, однако $C^{\infty}(\mathbf{R})$ вклалывается в $G$ канонически. Общая схема и понимание того, как правильно выбирать семейство методов регуляризации, позволили нам строить различные алгебры новых обо6-щенных функщий.

В частности, построены : алгебра, в которую влохены ультрараспределения; алгебра со всюду определенно сверткой и обратимым преобразованием Фурье, в которую вложено $S^{\prime \prime}(\mathbf{R})$ [7]; алгебры "новых обобщенцых функцин" с действующими в них интегральными преобразовағиями Лапласа, Мелтина, Ханкеля и др.[8]; алгебры периодических новых обобщенных функций [9], алгебра, построенная на базе пространств Соболева [10].

Все построения, упомянутые выше, своцятся по существу к факту, тто новая обобщенная функция является семейством гладких функций, зависящих от параметров. Функции, приналлехащие одному классу эквивалентности, не только имеют один и тот же предел, но также и один и тот же способ стремления $\mathrm{k}$ этому пределу. Поэтому с этой точки зрения мы мохем сказать, что новая обобщенная функция запомннает способ стремления к пределу, то есть она обладает памятъю. В связи с этим мы называем такие объекты "мнемофункциями" (от гредеского слова -память)

4. Мнемочасла Если алгебра $A$ содерхит единицу, то в фактор-алгебре $G=G_{M} / N$ существует подалгебра $\tilde{\mathbf{C}}$, состоящая из скалярных функий. Мы назовем эту алгебру алгеброй мнемодисел, а ее элементы назовем мнемочислами. Мнемочисла оказываются удобным аппаратом во многих высистениях. Это связано с тем фактом, что в $\tilde{\mathbf{C}}$ существуют бесконечно малые и бесконечно болышие числа

Пусть $E^{\prime}$-сопряженное к локаньно выпуклому пространству $E$ и $\langle f, \Psi\rangle-$ значение функционала $f$ на элементе $\Psi \in E$. Всякое $\tilde{\mathbf{C}}$-линеннное отобрахение из $E$ в $\tilde{\mathbf{C}}$ будем называтъ $\tilde{\mathbf{C}}-$ фунхционалом. Каждон мнемофункции $f(\phi, \tau)$ соответствует $\tilde{\mathbf{C}}$-функционал $\tilde{f}$ на $E$, определеннын формулой

$$
<\tilde{f}, \Psi>=f(\phi, \tau), \psi>, \quad \psi \in E .
$$

Мнемофункцию $f$ и злемент $u \in E^{\prime}$ называем ассоциированными, если $\langle f(\phi, \tau), \psi\rangle \longrightarrow\langle u, \psi\rangle$ по фильтру $F$ дия каждых $\phi \in \Phi$ и $\psi \in E$.

Отметим, что много разпнтых мнемофункцин мохет быть ассоциировано с одним элементом $u \in E^{\prime}$ и что $\tilde{\mathbf{C}}$-функционал $\tilde{f}$ не однозначно определяет мнемофункцию $f$. На первын взгляд это мохет казатъся недостатком теории. Однако, с другой стороны, эта неоднозначность является новым шагом к решению ряда проблем. Например, моделирование частицы посредством $\delta$ функции Дирака вполне приемлемо в линейных задачах. Однако такой подход является весьма грубым в нелинейном случае и здесь имеются определенные трудности. Чтобы их преодолеть, мы нуждаемся в дополнительной информации относительно объекта наштего рассмотрения. Такая информация приводит $\mathbf{k}$ необходимости моделирования частиц мнемофункциям, ассоциированными с $\delta$-функценћ.

5. Прамеры. 5.1. Пусть $\delta_{\phi}-$ образ $\delta$-функции при вложении $D^{\prime}(\mathbf{R})$ в $G$, где $\Phi$ - множество вида (2). Тогда $\delta_{\phi}=\frac{1}{\tau} \phi_{q}\left(\frac{x}{\tau}\right)$. Пусть также

$$
a_{q}=\int \phi_{q}^{2}(x) d x \neq 0, \quad \Psi_{q}(x)=\frac{1}{a_{q}} \phi_{q}^{2}(x), \quad \tilde{a}=\left(a_{q}\right) \in \tilde{\mathbf{C}}
$$

Последовательность $\Psi_{q}$ определяет вложение $D^{\prime}(\mathbf{R})$ в $G\left(C^{\infty}(\mathbf{R})\right.$ вкладывается при этом как векторнос пространство, но не как алгебра). Пусть $\delta_{\Psi}-$ образ $\delta$-функции при этом влохении. Тогда $\delta_{\psi}^{2}=\frac{\tilde{a}}{\tau} \delta_{\psi}$, т. е. $\delta^{2}$ имеет вид мнемофункции $\delta_{\psi}$ с бесконечно больщим козффициентом $\frac{\tilde{a}}{\tau}$ из $\tilde{\mathbf{C}}$. 
5.2. Умножение непрерывных функций не совпадает с умножением в $G$, когда $D^{\prime}(\mathbf{R})$ вложено в $G$. Однако оно отличается от умножения в $G$ только на бесконечно малые значения. Например, пусть $|x|_{\phi}$ - образ функции $|x|$ при вложении $\phi$. Тогда $|x|_{\phi}^{2} \neq x^{2}$, но $|x|_{\phi}^{2}-x^{2}=\tau^{3} c \delta_{n}$, где $c \in \tilde{\mathbf{C}}, \tau$. е. разность есть $-\delta$-функция с бесконеqно малым коэффициентом. Различие между новой теорией и классической может быть разъяснено на примере выражения $\left(|x|^{2}-x^{2}\right) \delta_{\phi}^{3}$. В класситеской теории $|x|^{2}-x^{2}=0$ и $\delta^{3}$ не определено. В новой же теории $\left(|x|^{2}-x^{2}\right) \delta_{\phi}^{3}=c \delta_{\psi}$, где с-некоторое мнемо- число.

Выписление $\tilde{\mathbf{C}}$-функционалов, ассоциированных с мнемофункциями мохет быг сведено к исследованию асимптотики интегралов, зависялцих от параметра. Бесусловно, что такие вычисления были сделаны намного ранее, чем была создана теория новьх обобщенных функций. Например, асимптотическое поведение $\delta$-образнон последовательности $\frac{1}{\pi} \frac{1}{x^{2}+\tau^{2}}$ проанапизировано в [12], то есть соответствующин $\tilde{\mathbf{C}}$-функционал оценен.

Подо6ные оценки используются в теории дифференциањњњх уравнений с малым параметром, теории сингулярных возмущений и т.П.. В общем, ситуадия слохившаяся после создания теории новых обобщенных функцин, напоминает ранее извествуғ в математическом "фольклоре". Л.Янг [13] пишет, что после появления книги Л.Шварца по теории распределений все математики разделились на три класса : первын класс образовали те, которые говорили, что они знают это лучше Шварца; во второй класс попали те, которые утверждали, что они знапи это рөњъше Шварца; третий хе класс состоял только из рецензента книги Шварца, который называл все это бессмыслицен!

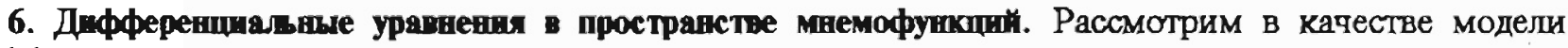
цифференциальное уравнение

$$
u^{\prime}=\delta u+\delta, \quad u(-1)=1
$$

Это уравнение не имеет решенй в пространстве непрерывных в нуле функцин. Если же функция $u$ разрыпна в нуле, то произведение $\delta u$ не определено. Это означает, что в классияеской теории обобщенных функци нет понятия решения этого уравненяя.

При рассмотрении уравнения (3) в пространстве мнемофунпаи необходимо выбрать некоторые мнемофункции, ассоциированные с $\delta$-функиией, например, $\delta_{\psi}$ и $\delta_{\phi}$ и исследовать уравнение

$$
u^{\prime}=\delta_{\phi} u+\delta_{\Psi}
$$

Решение этого уравнения ассоциируется с обыкновенной функцией видв

$$
u_{0}(x)= \begin{cases}1, & x \leq 0 \\ \mu, & x>0,\end{cases}
$$

где число $\mu$ зависит от выбора мнемофуккцин, которыцй мы сделали. Мы подчеркиваем, что информация относительно числа $\mu$ была полугена в результате выбора мнемофункцин, то есть в результате уточнения постановки задачи.

Даже в случае линейнља уравнений [14] типа

(5)

$$
u^{\prime}+a \cdot u=\delta_{\phi} \quad \text { где } a \text {-действитељьое число, }
$$

возникает вопрос уточнения постановкя задачи. Поскольку не шля всякон мнемофункции, ассоциирующейся с $\delta$-функией Дирака, будет существовать решение уравнения (5) в атебре мнемофункций, содержаще пространство распределений $S^{\prime}(\mathbf{R})$.

7. Спектральая теория оперяторов. Пусть $X$ - банахова алгебра с единицей $e$, в частности, алгебра $L(H)$-линейньгх ограниченньг операторов в банаховом пространстве $H$ и пусть $G_{M}(X)$ -атебра медленно (не быстрее некоторой стелени) растуших последователностей из $X$, а $N-$ идеал в $G_{M}$, состояший из быстро (быстрее любой степени) убывающих последовательностей из 
$X$. Тогда фактор-алтебру $G_{M} / N$ обозначим перез $X_{*}$, а соотнететующее ее кольио мнемочисел обояначим через $\mathbf{C}_{*}$.

Алгебра $X_{*}$ является $\mathbf{C}_{*}$-алгеброй, то есть модулем над кольцом $\mathbf{C}_{*}$ и, как выяснилось [14], естестрениая топология на ней ест неархимедова топология.

Если $\tilde{a} \in X_{*}$, то обобщенным спектром элемента $\tilde{a}$ назынается подмножество $\tilde{\sigma}(\tilde{a})$ из $\mathbf{C}_{*}$ вида $\tilde{\sigma}(\tilde{a})=\left\{\tilde{\lambda} \in \mathbf{C}_{*}: \tilde{\lambda} \tilde{e}-\tilde{a}-\right.$ не обратим в $\left.X_{*}\right\}$.

В работе [15] устаноилено, что $A_{*}$ является банаховой неархимедово нормброванной $\mathbf{C}_{*}-$

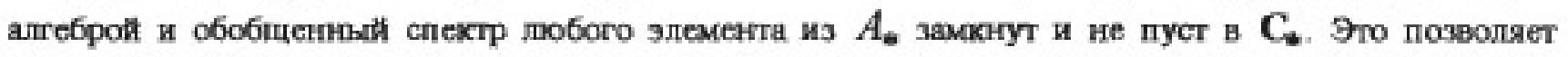
развит спектральную теорию элементов алгебры $A_{*}$ -

Элементы алгебры $L(H)$ естественно называть обобщенными операторами. Поскольку многие неораничеппые операторы мохио трактоват как обобщелпые, то этот подход позволяет по новому азлянут на многие вопроск слектрапьнй теории и фуикционатьного истисления для неограниченных операторов.

Прияедем пример. Рассмотрим последовательност ограниченных операторов в пространстве непре -рывньх фунстини $C[0,1]$.

$$
A_{n} x(t)=n x(t)-n^{2} \int_{0}^{t} x(s) e^{n(s-t)} d s
$$

Нормы таких операторов $\left\|A_{n}\right\|$ растут не быстрее некоторой степени $\boldsymbol{n}$. Последователыост операторов $A_{n}$ будет залават некоторьй элемент $\tilde{A}$ из $X_{*}$, если в качестве $X$ взят антебру ограниченньх опереторов в пространстве $C[0,1]$.

Пусть имесм оператор $A=d / d t$ осласыю определения

$$
D(A)=\left\{x(t) \in C[0,1] ; x^{\prime}(t) \in C[0,1], x(0)=0\right\} .
$$

Последовательность операторов

$$
A_{n} x(t)=n x(t)-n^{2} \int_{0}^{t} x(s) e^{n(s-t)} d s
$$

сищно сходится к оператору $A$, то ест обобщенпны оператор $A$ будет ассоциироватья с неораниченпам оператором $A$.

Спектр оператора $A$ пуст в $\mathbf{C}_{*}$, поскотку существование обрапного оператора к $A-\lambda I$ эквивалентно разрешимости задачи Коши

$$
\left\{\begin{array}{l}
u^{\prime}(t)-\lambda u(t)=f(t), \\
u(0)=0
\end{array}\right.
$$

Обобщенпы спектр $\tilde{A}$, в свою очеред, не пуст в кольне обобщенньх комплекснкх чисел $C_{\text {* }}$. И для обобщенного оператора $\tilde{A}$, порождаемого последовательностьо ограниченных операторов

$$
A_{n} x(t)=n x(t)-n^{2} \int_{0}^{t} x(s) e^{n(s-t)} d s
$$

который ассоциируетея с неограниченным оператором $A$ с пустым спектром, получено [15] следующе описание обобщенnого слектра $\bar{A}$.

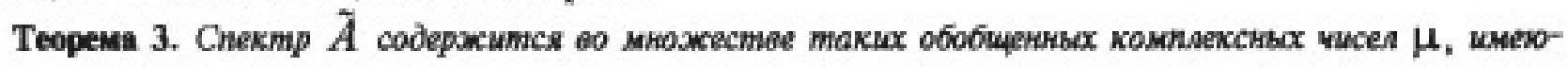

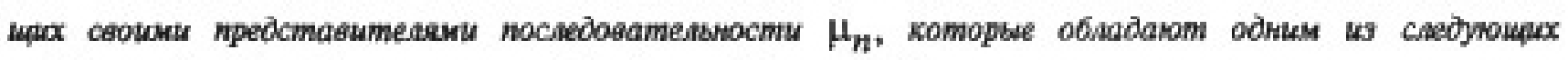

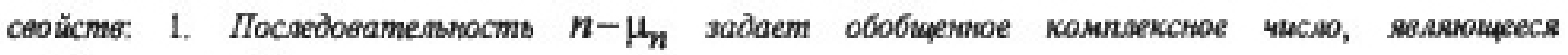
меобратимым \& $\mathbf{C}_{\text {* }}$.

2. Ilacoedoeamestocms $\mu_{n}$ makoea, umo 


$$
\left|\mu_{n}-\left(n-\frac{n^{2}}{2\left(n+c_{1}+c_{2} \ln n\right)}\right)\right|^{\prime} \leq \frac{n^{2}}{2\left(n+c_{1}+c_{2} \ln n\right)}, \quad c_{1}, c_{2}-\text { константы. }
$$

8. Алтебра ойобщенных случайных процессов. Параллельно с развитием теории обобщенных функций шло развитие теории обобщеипых случайиых процессов. Так на основе теории распреде-лений И.М.Гельфандом была создана теория обобшенных случайных процессов. КУрбаником прелложена своя трактовка обобщенных случайных процессов, опирающаяся на секвендиальный подхоп Я Микусинского. Нало сказать, что несмотря на значимость данғыг теорий в современной теории случайных процессов, они не нашли широкого испољьования в дифферешиальных уравнениях со случайными функциями в силу неприменимосги их для решения нелинейных задач.

В работах [16],[17] на осиове теории мнемофункций предложена конструкция алгебры обобще-нных случайных процессов. Рассмотрим наиболее простой случай алгебры мнемофункций Ю.B. Егорова.

Пусть $T=[0, a] \subset \mathbf{R}, \quad(\Omega, \mathrm{A}, P)$-полное вероятностное пространств. Рассмотрим множество последователыностей случайных функий $f_{n}: T \times \Omega \rightarrow \mathbf{R}, \quad G_{M}(T, \Omega)=\left\{\left(f_{n}(t, \omega)\right): f_{n}(t, \omega)-\right.$ случайная величина при фиксированных $n$ и $t ; f_{n}(t, \omega) \in C^{\infty}(T)$ для почти всех $\left.\omega \in \Omega\right\}$ Выделим в $G_{M}(T, \Omega)$ идеал $N(T, \Omega)=\left\{\left(f_{n}\right) \in G_{M}(T, \Omega):\right.$ существует $n_{0}$, что дия любого $t \in T$ и. лля любого $\boldsymbol{n} \geq n_{0}$ $f_{n}(t, \omega)=0$ घля почти всех $\left.\omega \in \Omega\right\}$

Aлrебру $G(T, \Omega)=G_{M}(T, \Omega) / N(T, \Omega)$ назовем алтеброй случайныгх мнемопроцессов.

Далее, пусть $\check{\mathbf{R}}$ - расширенная прямая по Егорову, а

$$
\tilde{T}=\left\{\tilde{t}=\left[\left(t_{n}\right)\right] \in \tilde{\mathbf{R}}: \forall\left(t_{n}\right) \in \tilde{t}, 0 \leq \tilde{t} \leq a, n=1,2, \ldots\right\} \text {. }
$$

Через $G(\tilde{T}, \Omega)$ обозначим алгебрусслучайньгх мнемофункцини вида $\tilde{F}(\tilde{t}, \omega)=\left[\left(f_{n}\left(t_{n}, \omega\right)\right)\right]$, где $\tilde{t}=\left[\left(t_{n}\right)\right] \in \tilde{T},\left[\left(f_{n}\left(t_{n}, \omega\right)\right)\right] \in G(T, \Omega)$ для любого $t \in T$.

Алгебу $G(\tilde{T}, \Omega)$ такхе назовем алгеброй случайных мнемопроцессов. Заметим, что конструкция апгебры новая и для неслучайного анализа.

В работе [17] на основе аппарата алтебр случайнырх мнемопроцессов предложен единый подход исследования всех известных классов дифференщиальных уравнении со случан̆ными функциями. Этот подход базируется на понятии обобщенного стохастического дифференциала в $G(\tilde{T} ; \Omega)$.

Пусть

$$
H=\left\{\tilde{h}=\left[\left(h_{n}\right)\right] \in \tilde{\mathbf{R}} \backslash\{0\}: \lim _{n \rightarrow \infty} h_{n}=0\right\} .
$$

Тогда положим по определению

$$
d_{\tilde{h}}=\tilde{F}(\tilde{h}, \omega)=\left[\left(f_{n}\left(t+h_{n}, \omega\right)-f_{n}(t, \omega)\right)\right], \text { где } \tilde{h}=\left[\left(h_{n}\right)\right] \in H, \tilde{t}+\tilde{h} \in \tilde{T}
$$

Выделим во множестве $H$ следующие подмножеств:

$$
\begin{aligned}
& S=\left\{\tilde{h} \in H: h_{n}=0(1 / n), n \rightarrow \infty\right\} \text {-область Стратановича ; } \\
& I=\left\{\tilde{h} \in H:(1 / n)=0\left(h_{n}\right), n \rightarrow \infty\right\} \text {-область Ито. }
\end{aligned}
$$

Известно, что дифференциатьные уравнения, содержацие в правой части обобшенные случайные процессы типа "белого шума" невозможно исследовать классическими методами теории обыкновенных дифференциалыных уравнений. Для этого К Ито была специатьно разработана теория стохастических пифференщилльных уравненин, базиругщаяся на понятиях стохастических интегралов Ито и Стратановича. В работе [18] показано, что решения стохастических дифференциальньх уравнений Ито и Стратановича́ могут быгь аппроксимированы решцениями уравненин в 
дифференциалах в ашебре $G(\tilde{T}, \Omega)$. Методика доказательства этих утверждени опирается на ионятия обобшенного стохастического дифференциала и областей Ито и Стратановича.

В рабоге [19] пгозано, чго данные методы могут быть успешно применены для исследования решеняй новых кгассов нелинейных цифференгиальньх уравнений со случайными функциями.

\section{Jnreparypa}

1. L. Schwarz. Sur l'impossibilite' de la multiplication des distributions. C.R Acad sci. Paris. 1954, v.239, $874-848$.

2. Ю. В Егоров. О теории обобщеннњг функциц. Успехи мат. наук, 1990, т.45, N.5 (275), 3 - 40.

3. Хр. Христов, Б. П. Дамянов. Асимптотические функтии -Новый класс обобщенниг функцин. Bulgar. J.Phys. ч. 1, 1978, N.6, 543 - 556, ч. II, 1979, N.1, 3 - 23, ч. III, 1979, N.3, 245 - 256, ч. IV, 1979, N.4, $377-397$.

4. В. К Иванов. Умножение распределений и регуляризация расходящихся интегралов. Известия ВУЗов, Мaт., 1971, N.1, $41-49$.

5. J. F. Colombeau. New generalized functions and multiplication of distributions. North Holland, 1989. 